\title{
The Double STARS Research Seminar: An analysis of its effects and methodologies
}

\author{
Alexander Beltzer-Sweeney ${ }^{1,3}$, Stephen L. White $2,3 *$
}

\begin{abstract}
The Double STARS (STEM Through Astronomy Research for Students) seminar is a hybrid online/in-person research experience aiming to encourage critical thinking and data analysis through observational astronomy via robotic telescopes. Presented here is a program analysis from the perspective of the authors, two undergraduate students who have previously participated in the program, who are now mentoring students in both California and New Mexico. Data collected from 57 past students, both online and in-person, are presented in order to give a broader understanding of the successes and challenges the program has faced. This paper provides the education community with valuable knowledge of how similar programs can be adapted to best suit the needs of students as well as the ways in which programs such as these may help students in the areas of professional development, research, and overall scientific understanding.

\section{Keywords}

Student Research - Double Stars - Robotic Telescopes

${ }^{1}$ Undergraduate Student, Department of Physics and Astronomy, San Diego Mesa College

${ }^{2}$ Undergraduate Student, Department of Physics and Mathematics, New Mexico Institute of Mining and

Technology

${ }^{3}$ Student Research Advisor, Boyce Research Initiatives and Education Foundation, San Diego, CA

${ }^{*}$ Corresponding author: Stephen.White@student.nmt.edu
\end{abstract}

\section{Introduction}

The authors both started out as students of the Double STARS (STEM Through Astronomy Research for Students) seminar (Boyce and Boyce 2017) during the Fall of 2016 and Spring of 2017, respectively, at community colleges in San Diego, California, and have subsequently moved on to mentor their peers at San Diego Mesa College and the New Mexico Institute of Mining and Technology. After publishing multiple papers dealing with the astrometry of double star systems (see Korat et al. 2017; White et al. 2018) on the authors became interested in the questions of exactly how effective these pro- grams were in developing an understanding of the scientific process, and how the Double STARS seminar could be shaped to maximally affect the students involved with it.

This paper aims to provide the education community with the perspective of the students involved in the Double STARS seminar (hereafter, the seminar), as well as the ways in which the program has helped students in the areas of professional development and overall scientific understanding. Section 2 will briefly describe the background of the seminar. Section 3 will give an overview of the survey conducted, and how it was implemented. Section 4 will take an in depth look at the methodologies of the seminar, and discuss the positive and negative short-term affects for the students involved, utiliz- 
ing the results from the survey. While no long term effects are yet measured, we believe the survey and anecdotal evidence provided will be of value to the educational community.

\section{Background of the DoubleSTARS Seminar}

The first iteration of the seminar-implemented by Boyce-Astro, the observational astronomy program within BRIEF (Boyce Research Initiatives and Education Foundation) — came to fruition in the Fall of 2016 after Pat and Grady Boyce spent two years alongside Russ Genet ( e.g. Johnson et al. 2015) developing the structure and curriculum of the program (Boyce and Boyce 2017). The seminar would eventually evolve into a hybrid online/in-person program allowing its reach to become far greater than if it were limited to only formal classrooms. Following the first successful semester in the Fall of 2016, two more seminars were added to the program to encompass a wider breadth of the field, and to allow the students who successfully completed the first semester to continue on to more advanced and independent research.

The STARS (STEM Through Astronomy Research for Students) seminars, as they became known, are divided into three separate portions as follows:

- Double STARS: a first semester seminar based around the astrometry of double star systems

- Variable STARS: a second semester seminar based around both single-image and time series photometry

- Advanced STARS: guided independent research in stellar astronomy, exoplanets, and asteroids.

\section{The Survey}

Google Forms was utilized in order to conduct the survey, and consisted of multiple-choice, Likert scale and open-ended questions. Some typical questions are provided in Figure 1. This survey was sent to every single student that had taken part in the seminar from the Spring 2016 to the Spring 2018. A fairly even distribution of respondents from each of the last 4 semesters was received; with exception of the first semester the program was offered. The distribution is shown in Figure 2. Approximately $31 \%$, or 57 , of those participants responded over the course of about 5 weeks.

The first section of the survey aimed to gain a better understanding of the demographics of the respondents. The broad results are presented in Figure 3. As was expected, most students who had decided on a major were focusing on the STEM fields. The undecided $23 \%$ is composed of mostly high school students for which no major has been declared. There were 10 different fields represented by the respondents. It is notable that a program without specific recruiting guidelines represents such a diverse population of students.

The seminar works with people from many different levels of education. As seen in Figure 4, most of the participants of the program were either High School students, or in some sort of higher education. It was only in the last semester surveyed that the program was first trialled at a four-year college, New Mexico Tech, where one the authors (SW) took the program after transferring from San Diego Mesa College. While the students here were very capable of completing the project, there was some difficulty keeping the interest of the junior level participants because the author was already knowledgeable in the aspects the seminar covered. It may be best for entry-level programs similar to the Double STARS seminar to focus on high schools and community colleges because of the difficulty in keeping higher education level participants interested and engaged in all aspects of the program while utilizing one core curriculum for all students involved.

Finally, the gender breakdown of the survey participants was analyzed. Out of the 57 total respondents, $49 \%$ were female and $51 \%$ were male. The demographic of the survey respondents broadly mirrored the breakdown of students who have taken the seminar, which is $52 \%$ male and $48 \%$ female. The seminar has been adopted by other educational programs in the San Diego area, such as the Better Education for Women in Science and Engineering 


\section{$\leftarrow$ DoublestaRs ${ }^{\mathrm{Tn}}$ Evaluation Survey $\hat{a}$}

QUESTIONS

RESPONSES

\section{7}

How helpful was the external review from supervisors and the Boyces in improving your team's paper?
1
2
3
4
5
Not at all helpful
$\bigcirc \bigcirc$ Very helpful

Roughly, how well did you understand the particular aspect of astronomy you * studied after completing the seminar?

$\begin{array}{lllll}1 & 2 & 3 & 4 & 5\end{array}$

Not well

$0=0$

How well did the proposal presentation help to facilitate the development of your project?
1
2
3
4
5
Not well

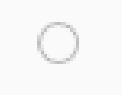
$\bigcirc$

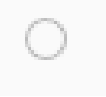
Very well

How difficult was it for your team to write up its paper? *

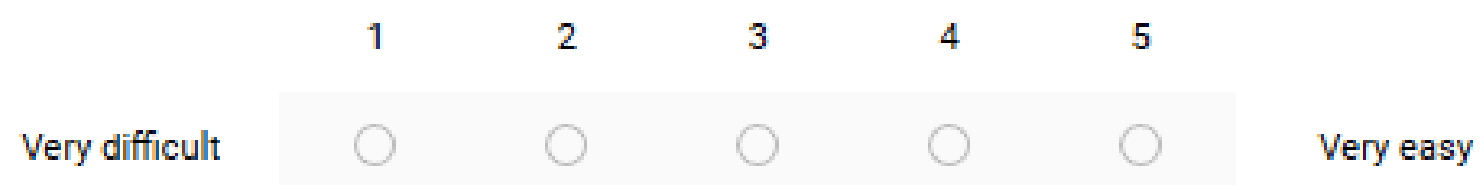

Figure 1. An example of the survey distributed to participants of the seminar.

— better known as BeWise — contributed slightly to the demographics of the survey respondents and 


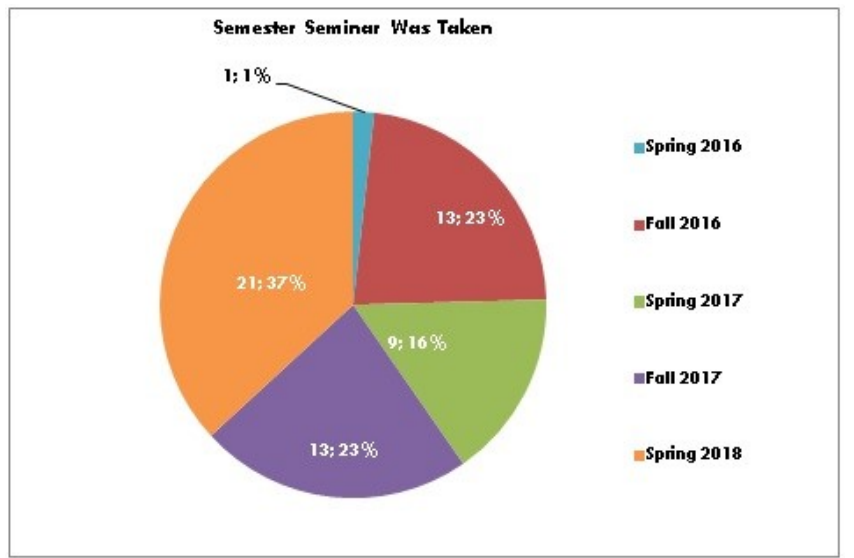

Figure 2. Semester that survey respondents took the seminar.

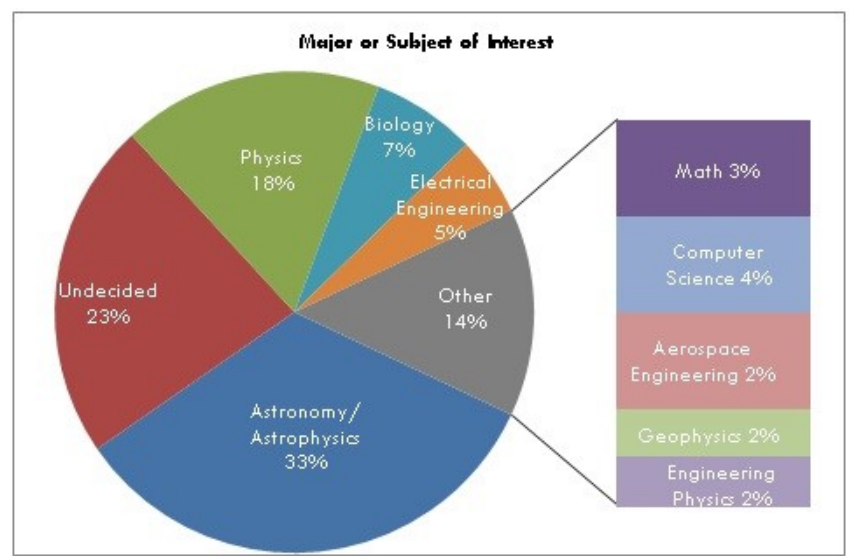

Figure 3. Major or subject of interest of survey participants.

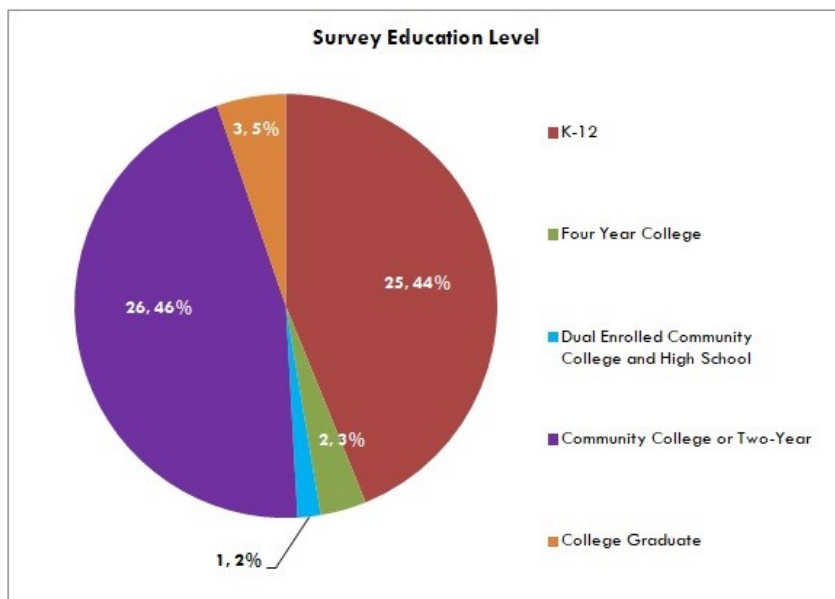

Figure 4. Education level of the survey participant when taking the seminar.

to the program as a whole.

\section{Methodologies and Their Effects}

The seminar takes place over 16 weeks, and coincides with the Spring or Fall semester for most students. This time period allows for the students to have enough time to: learn the material, develop and present a proposal presentation, acquire test and final images, analyze their images, present what they found at a final presentation with their fellow researchers, and draft their final paper.

The students are provided a syllabus which lists assignments and deliverables for each week at the beginning of the semester, and links to online web content. An example of a typical weekly tasks is shown in Figure 5. For students taking the online version of the seminar, weekly Zoom meetings are held to review assigned materials and cover any other concerns brought up by students. The online meetings, typically an hour long, are open to all students taking the course during the semester, and any participants with questions or concerns are encouraged to join in.

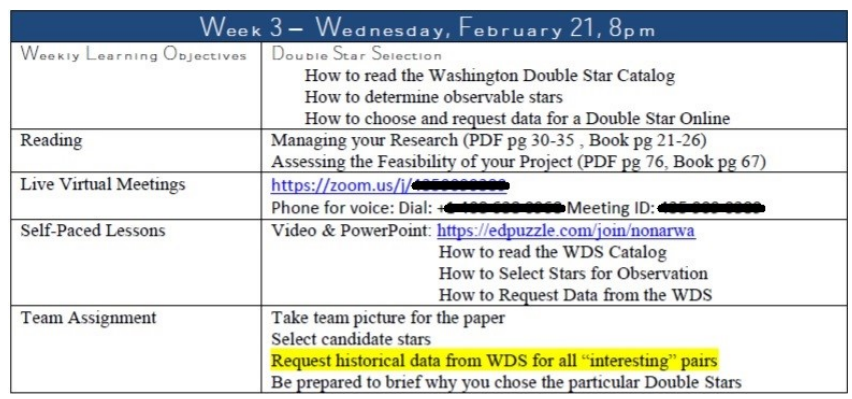

Figure 5. Example week from the DoubleSTARS Seminar syllabus

The syllabus lists out all of the required readings from Russ Genet's Small Telescope Astronomical Research (STAR) Handbook (Genet et al. 2015), shown in Figure 6, as well as the Edpuzzle videos created by Grady Boyce. The STAR Handbook is provided to the students as a PDF, and is an invaluable tool for learning the intricacies required in portions of the research. Edpuzzle is utilized to distribute self-paced video lectures, and uniquely allows questions during playback to ensure comprehension of the subject at hand. Students have access to a library of video content that covers every aspect of the course. 

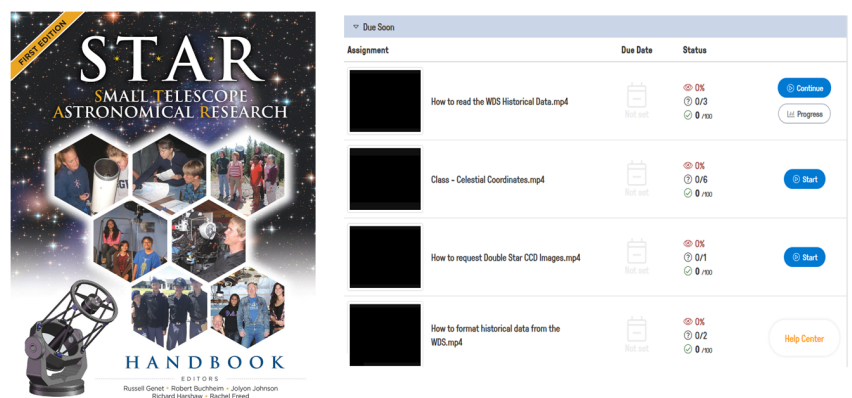

Figure 6. Russ Genet's STAR Handbook (left), and an example of the EdPuzzle webpage (right).

The seminar can be broken down into 7 main milestone points:

- Week 1-2: Team selection and role assignment

- Week 3-5: System selection and historical research

- Week 6: Proposal presentation and test images

- Week 7: Order final observations

- Week 7-15: Data analysis, initial drafts, final presentation preparation

- Week 15: Final presentation

- Week 16: Final draft (expected) due date

These milestone points were utilized as the methodologies of the seminar, and each one will be considered individually along with supporting data from the survey, where available, to express the effects they have on the students involved.

\section{Week 1-2: Team Selection and Role Assign- ment}

During the first week students are provided with the course materials, introduced to their teammates, and role assignments are chosen. Team selection processes vary depending on who is teaching the program. For example, at Mesa College where the program is taught by astronomy professor Dr. Irena Stojimirovic, students are split into teams based on their skills, strengths, and willingness to take on the position. Team leadership is chosen among teams and is not always decided during these first two weeks. Regardless of the roles taken on, all students are required to learn the same material in order to communicate effectively amongst themselves, and in order to provide a failsafe in the case of a student being unable to complete their assigned task or in the event of an unexpected life circumstance arising.

Almost half of the survey respondents had interdisciplinary skills that supported their team and the requirements of the project. The broad distribution of reported unique talents is presented in Figure 7. Some students indicated that they did not have any applicable skills, and that they wished that they had a unique talent that helped out the team. In these students' specific survey responses, they said that, despite lacking what they considered to be relevant skills, the program motivated them to continue within the STEM field, and that this was an experience different from any other they have had in a school class. It is anecdotally reported by project personnel it is often the students who feel the most under-prepared for this type of research that come up with unique, inventive methodologies and solutions to the problems faced within the seminar.

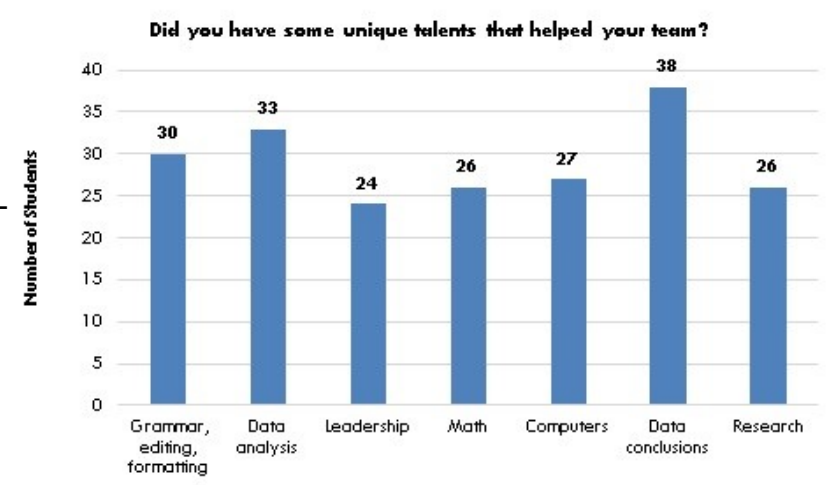

Figure 7. Survey question regarding individual talents of the respondent that helped them contribute to their team.

This question allowed "other" responses, and some of these responses were:

- "I am good with computers so was able to contribute in technical areas; I wish I could've helped more..." 
- "I am able to pick up the pace where others left off “

Like with any team-based project, there are varying levels of participation, as shown in Figure 8. A majority of the respondents noted that there was some lack of involvement by at least one of their teammates. Roughly half of that majority said that this was not an issue for them; while the others stated that this was a concern, and that they should not have been included as coauthors. This lack of participation from select students has been observed while involved with this program as mentors, and it is often an issue that there seems to be no clear answers for. The issue lies in that it has not been possible to properly motivate all of the students who are not passionate about conducting the research.

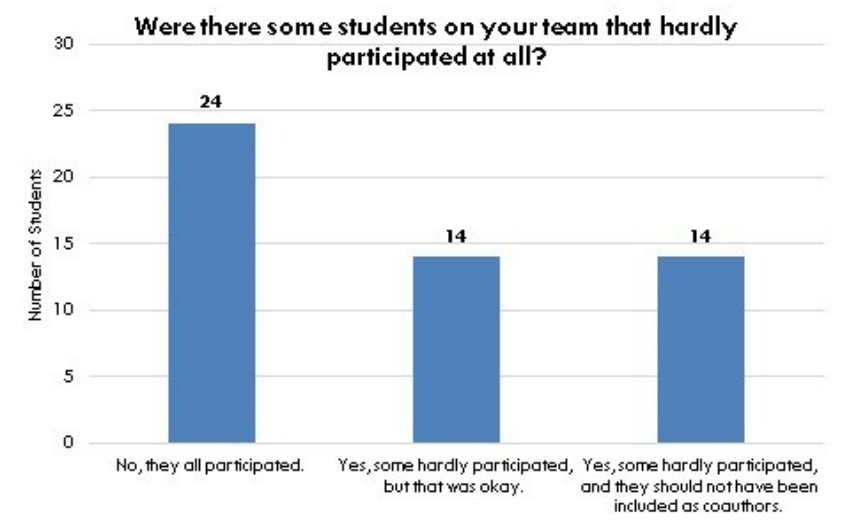

Figure 8. Survey question regarding the participation of team members.

\section{Week 3-5: System Selection and Historical Research}

During weeks 3-5, the students are asked to explore the Washington Double Star Catalog (Mason et al. 2010) in order to find a system that both lies inside of certain pre-set parameters and that they find interesting and worthy of research. This is often a part of the seminar that is reported as most daunting for students, as they have only just figured out what double stars are, and yet they are asked to look at massive lists of data in an attempt to find their systems using qualitative analysis as opposed to using strict quantitative guidelines. As of the Fall
2018 semester of the program, the ways in which students look for potential double star systems to study has been updated. The students now utilize a spreadsheet of in depth astrometric data (Harshaw 2018) from the Gaia data release 1 and data release 2 (Gaia et al. 2018).

After selecting a small number of potential systems, historical data is requested from the US Naval Observatory, and the students look at multiple online resources while waiting for that data to be returned to them. Typically, students utilize resources such as StelleDoppie, Simbad, and now the Gaia data release 1 and 2 in order to find existing data about the system. The students are also encouraged to dig into old publications about their systems, and begin to form the introduction to their own papers at this time. The students' introductions typically involve a brief outlining of what the different types of double star systems are, the historical data found on their system, and the initial discoverer of the double star system - all of which will also be used in their upcoming proposal presentations.

\section{Week 6: Proposal Presentation and Test Images}

The proposal presentation in the seminar provides students with a few different opportunities rolled into one single event. The purpose of the presentation is for the students to have a venue to demonstrate what they have learned, the historical research they have performed, and the candidate systems they have chosen for their project. The students are required to show, at minimum, basic information about the stars, what telescope system and possible filters they will use to image with, a Gantt chart with their timeline for the semester, and why they find this system interesting for observations. All of that information is necessary when writing up their paper towards the end of the semester. This experience also gives students a chance to speak in front of a large group, sometimes for the first time, and provides them with valuable feedback concerning the systems they chose.

While some students might find talking in front of a large group to be stressful, the proposal presentation greatly assisted them in the development of 
their project. The general distributions of answers to this question are presented in Figure 9. Only one student said that this process provided no benefit to them. In a previous semester, students were required to not only present this information, but write it out similar to how scientists would write a grant proposal. They were required to apply for telescope time, and the groups with the best proposals were given extra time for their observations. This system could be utilized for all semesters, as it is nearest to the reality of scientific research, and students would potentially take the observation period more seriously.

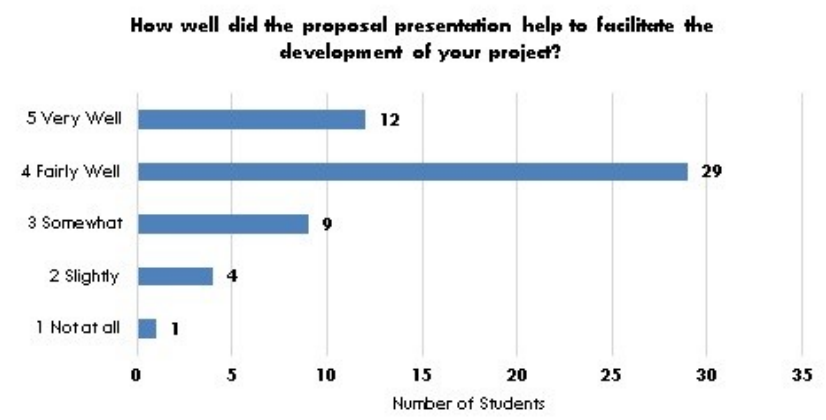

Figure 9. Survey question regarding the usefulness of the seminar proposal presentation.

The students take test images to confirm that the exposure lengths and filters they chose are ideal for imaging their selected system. During the first two semesters, Grady Boyce was responsible for ordering images through iTelescope (Boyce et al. 2016), with that responsibility being passed onto the student mentors in the Fall of 2017. In the Spring 2018 semester, BRIEF began working with the Las Cumbres Observatory (LCO) (Brown et al. 2013), and students were then trained on how to request data using that system. Utilizing the LCO telescopes and the Our Solar Siblings (OSS) pipeline (Fitzgerald 2018) freed up students to concentrate more on analyzing their data and writing their papers. This is because of the ease with which the images can be ordered through LCO, with the images being platesolved and "ready-to-use" once they receive them from the OSS pipeline.

This final week of preparation for the proposal and test imaging before going into the "real" data collection helps students by allowing them to plan, test, and receive back their images before submitting their final observation requests. The test image process allows them to adjust for any issues in their original plans before moving on to taking the science images.

\section{Week 7: Ordering Final Observations}

After taking their test images and reviewing them to make sure the exposure times and filter selections they chose will work, the students then move on to taking their final images. This process typically takes around 1-2 weeks from the time they take their images until they are available for students to begin analysis on. During this time period, students are encouraged to begin planning out the latter portions of their paper, and to prepare for image analysis and data collection. The distribution of responses, presented in Figure 10, shows that over three quarters of the respondents found the amount of time for imaging to be sufficient. We believe this is largely in part to the implementation of taking test images before simply jumping in and taking images for science purposes.

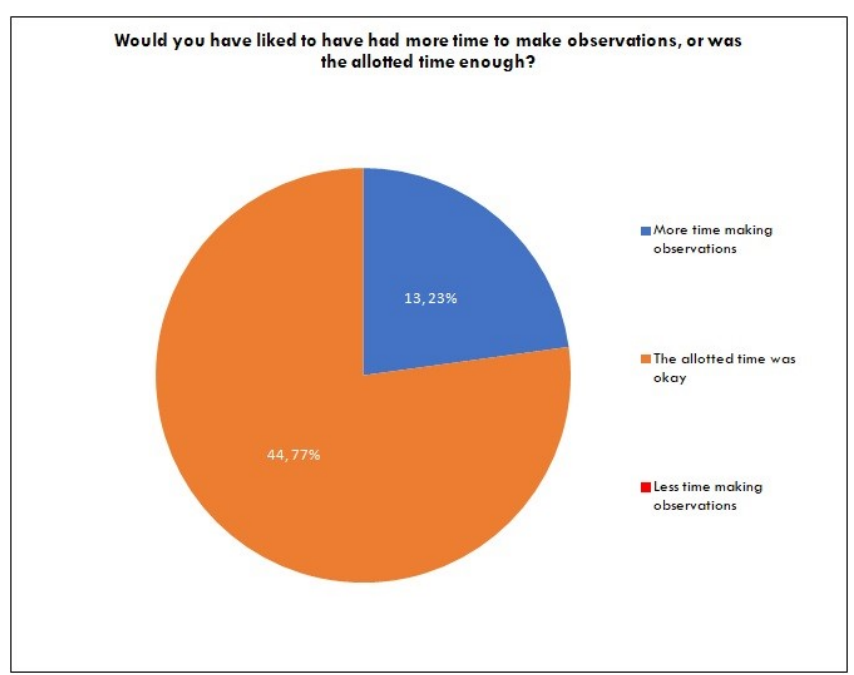

Figure 10. Survey question regarding the time allotment for observations during the seminar. No respondents thought there should be less time allocated for observations.

\section{Week 7-15: Data Analysis, Initial Drafts, and Final Presentation Preparation}

Over the next eight weeks students move on to data collection and analysis, start to form their initial 
drafts based off the work done so far and begin preparing for their final presentation. Over this time period, there are still scheduled group meetings, but they are now more focused on specific issues that might arise within each team.

When asked whether there should be prerequisites in order to take the seminar, the overwhelming response was no. The distribution of responses is shown in Figure 11. One respondent stated that, "I don't think the prerequisites were necessary because the instructors did a very good job of explaining everything we needed to know to complete this project." The one prerequisite that is asked by Boyce-Astro is a desire to contribute and participate in the process. Students have come into this program with next to no practical experience in astronomy, and walked away with a new insight into what interests them and what their capabilities as a student are.

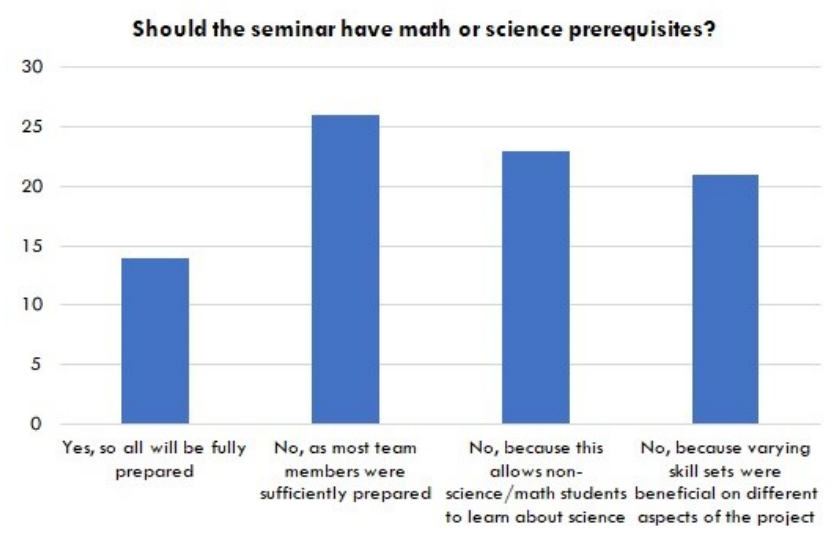

Figure 11. Survey question regarding whether or not the program should have math and/or science prerequisites.

This question allowed "other" responses, and some of these responses were:

- "The prerequisites should focus on character, work ethic, and analytical ability."

- "Prerequisites should not be required but should be suggested."

- "People with very little science pre-requisites should be vetted to ensure their motivations are in-line with the seminar."
As mentioned previously, the seminar requires students to develop a Gantt chart in order to keep their project on schedule. Having students develop this chart helps them maintain a rough timeline of events and deliverables, and also helps them learn how to properly allocate their time. The data around students' input on whether they were given enough time to form a well-written paper is presented in Figure 12. Overwhelmingly, the students believed they were given enough time to at least write a basic scientific paper, with only one student responding that there was not enough time to do a good job writing the paper.

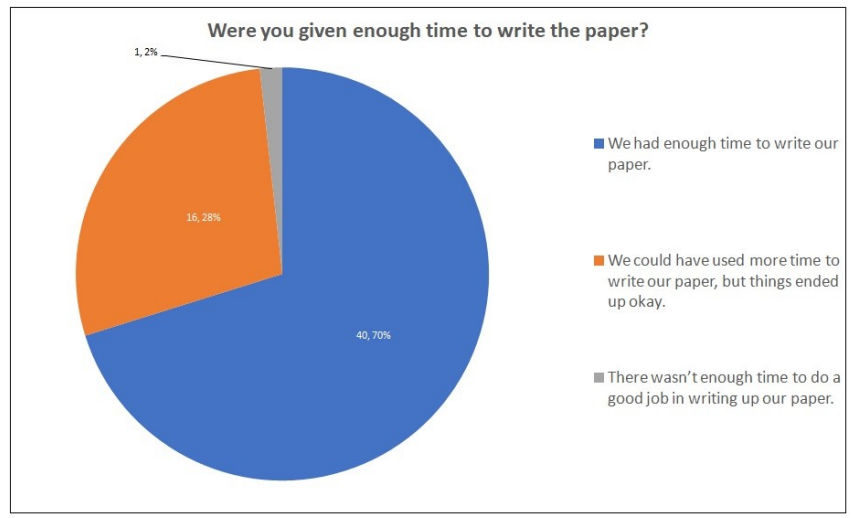

Figure 12. Survey question regarding the amount of time allotted to writing their paper.

One of the greatest benefits of a program being designed specifically for those new to the field is these students being able to collaborate with professional astronomers in undertaking their projects. When asked if they felt like they were immersed within a supportive professional-amateur community, $49 \%$ of the students indicated that they felt only somewhat immersed, with a single response stating that there was no immersion. The results for this question are represented in Figure 13. An as yet unanswered question for future research could be "What could make more students feel as if they were being supported more than they are currently?"

Students, be it in a program like this or in any other class situation, often feel a level of intimidation from their instructors. While some students feel comfortable engaging with their teachers, others might not, and as a result, will not communicate as much as others. The introduction of Peer Advi- 


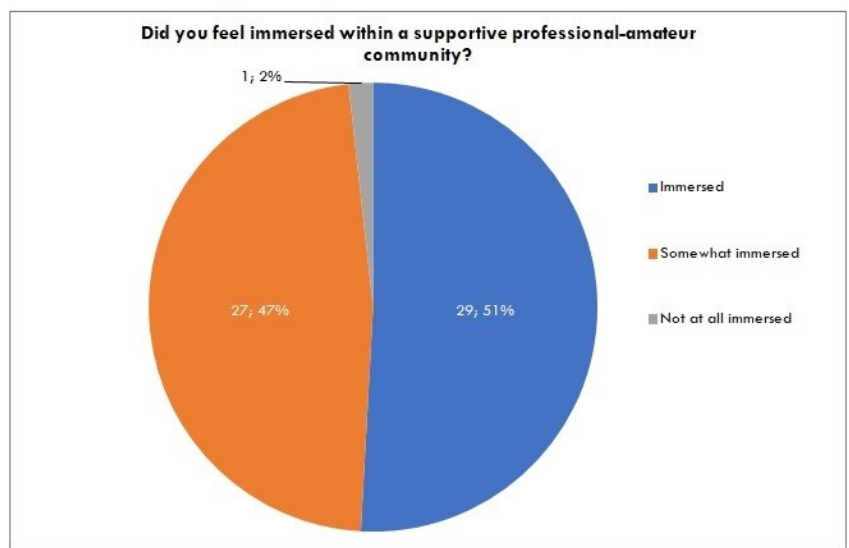

Figure 13. Survey question regarding the feeling of immersion within a professional-amateur community.

sors into the DoubleSTARS seminar allows students to reach out to someone who may not be as intimidating and busy as their instructor.

Conference attendance, as experienced by the authors and other participants, give students a valuable opportunity to interact with professionals in a way that is currently not done in the DoubleSTARS program. The students' successes and accomplishments in this field are largely due to their attendance, participation, and networking opportunities at local and international conferences. Attending these conferences allows students to gain experience answering tough questions that were posed by those viewing their posters and presentations, and also to see what kinds of research was being done outside of the narrow field of view they currently have.

\section{Week 15: Final Presentation}

During the final two weeks, drafts are ready to start going through peer review, if they have not already started, and the teams prepare their final presentation. The final presentation allows the students to show off their research to the same group of students and instructors as during the proposal presentation, and gives students a chance for final feedback regarding the methods they employed and conclusions they have drawn.

What students were taking away from the program after completion was explored in the survey, and the results from those questioned, presented in Figure 14, were quite encouraging. Out of the 57 people surveyed, 50 of them left this course feeling like they better understood the double star field than when they began, and not a single person reported zero post-completion understanding.

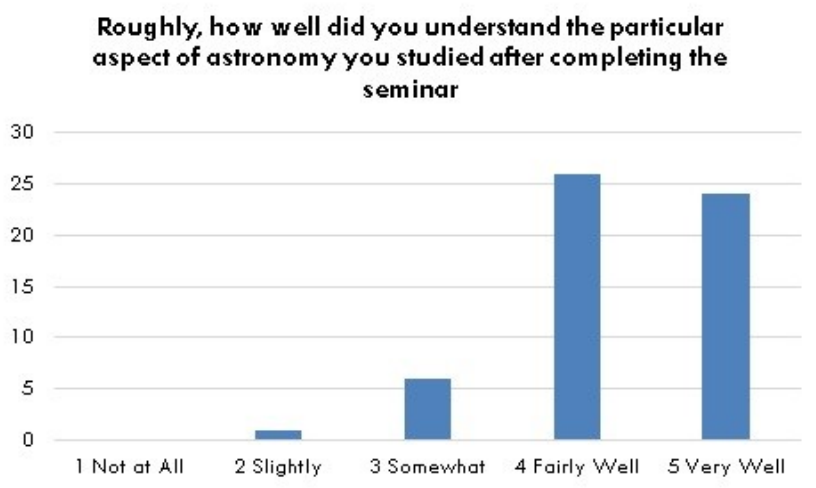

Figure 14. Survey question regarding the students' post research understanding of the field of double stars.

As discussed earlier, when dealing with how the authorship order is decided, the level of participation is taken into consideration by the group. The results from this aspect of the survey are presented in Figure 15. There are circumstances in most semesters where a student stops attending the program or participating with their team, and that student will generally either be put further down on the authorship order, or removed entirely and added to the acknowledgments depending on the amount of work contributed.

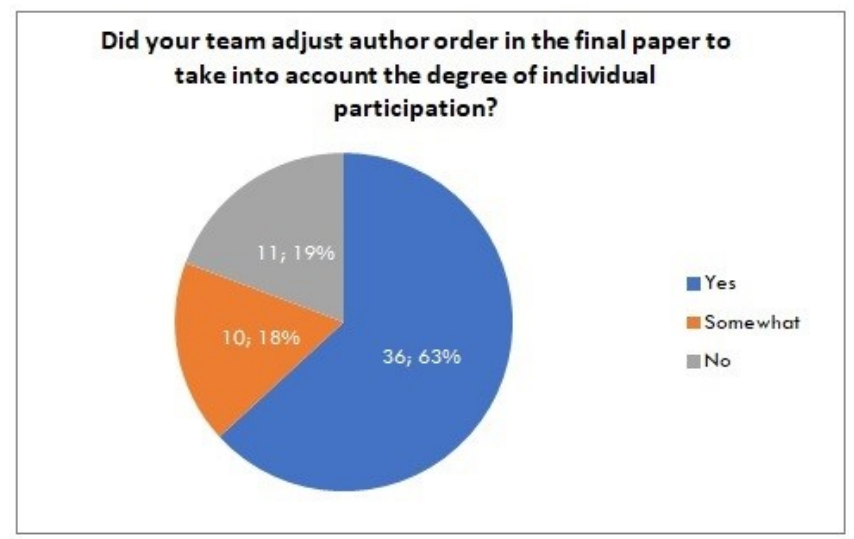

Figure 15. Survey question regarding the final author order and degree of individual participation. 


\section{Week 16: Final Draft (expected) Due Date}

In the final week of the seminar, and after the students have completed their final presentations, the final draft of the paper the students have been working on is expected to be turned in for peer review. Of course, peer review comes with more revisions, and these are handled via email. Students were able to come away from this class having learned more about the field of double star astrometry.Of the students $95 \%$, or 54 of them, reported that they felt this class was more than just an academic exercise, and that they were able to contribute to the scientific community at least in a minor way. The responses to this question are presented in Figure 16.

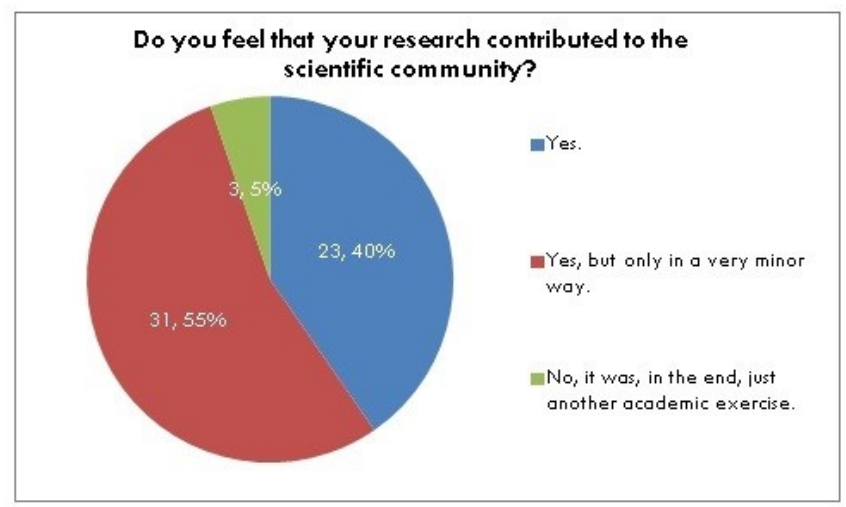

Figure 16. Survey question regarding individual contribution to the scientific community.

\section{Conclusion}

Students often feeling like the work they are doing is not "real science," or like they're not truly contributing to the scientific community. Admittedly, the authors themselves had these thoughts when we were on the student side of this program. This "imposter syndrome" and/or the feeling that students are "not doing real science" is an issue that was brought up multiple times in discussions at the RTSRE conferences, and is an issue that the educational community must face head on. The reality is that programs such as the DoubleSTARS seminar are not intended to be professional grade research programs performing cutting edge research. What these programs are intended to do is inspire early students to continue on in STEM related fields, as well as teaching them the basic skill sets that they will utilize throughout their early careers in a multitude of fields. More so now than ever before, the acquisition and sharing of data is an essential part of astronomy and the broader scientific community, and teaching students the methods that are utilized by scientists in every field across the board will allow them to make great leaps in their future careers.

Time and again, the work done with this seminar leads students to continue pursuing research careers, and accepting admission and scholarships to a multitude of colleges where they utilize the skills they obtained early on (e.g. Freed 2018, 2019a,b). This is something that has been expressed by our students, and is perfectly summarized by them when they were asked if there were any statements about the program they would like to make anonymously.

\begin{abstract}
"The whole seminar was so phenomenal. It gave me a better appreciation for research and has since led to a new career in the biotech industry for myself as I am still pursuing my degree. Thank you Boyce-Astro and Jae [Calanog] for giving students a chance to prove themselves and to work with others who are working towards the same goal!"

"I highly value the experience I gained by participating in this project, including skills such as analyzing data, working on a research team, and writing a professional-level research paper."
\end{abstract}

\section{Acknowledgments}

The authors would like to thank the organizers of the RTSRE conference, as well as Rachel Freed and Michael Fitzgerald for your invaluable advice, conversations, and guidance-without which this project would not have been possible. The authors would also like to thank Pat and Grady Boyce for the innumerable opportunities they have provided to us, and for their dedication to the advancement of student research in the astronomy field. 
The authors would like to acknowledge funding provided by the San Diego Mesa College STEM Scholars Grant. In addition, funding was also provided by The Department of Physics (Dr. Richard Sonnenfeld), The Department of Student-University Relations (Melissa Jaramillo-Flemming), and The Research and Economic Development Division (Dr. Van Romero) at the New Mexico Institute of Mining and Technology.

The authors would also like to acknowledge funding from the National Science Foundation-NSF Grant 1610350.

\section{References}

Boyce, P. and Boyce, G. (2017). A CommunityCentered Astronomy Research Program. In Society for Astronomical Sciences Annual Symposium, volume 36, pages 107-122.

Boyce, P., Boyce, G., Genet, R. M., Al-Zaben, F., Li, D., Li, Y., Dennis, A., Cao, Z., Li, J., Qu, S., et al. (2016). Measuring Visual Double Stars with Robotic Telescopes. AAS, 228:214-01.

Brown, T., Baliber, N., Bianco, F., Bowman, M., Burleson, B., Conway, P., Crellin, M., Depagne, É., De Vera, J., Dilday, B., et al. (2013). Las Cumbres Observatory global telescope network. Publications of the Astronomical Society of the Pacific, 125(931):1031.

Fitzgerald, M. (2018). The Our Solar Siblings Pipeline: Tackling the data issues of the scaling problem for robotic telescope based astronomy education projects.

Freed, R. (2018). The Astronomy Research Seminar: The Wide-Ranging Impact on Student Education and Careers. Preliminary Results. Society for Astronomical Sciences, 37:161-173.

Freed, R. (2019a). Astronomy Research Seminar Expansion and Building a Community-ofPractice. RTSRE Proceedings, 2(1).

Freed, R. (2019b). Evaluation of the Astronomy Research Seminar. RTSRE Proceedings, 2(1).
Gaia, C., Brown, A., Vallenari, A., Prusti, T., de Bruijne, J., Babusiaux, C., Juhász, Á., Marschalkó, G., Marton, G., Molnár, L., et al. (2018). Gaia Data Release 2 Summary of the contents and survey properties. Astronomy \& Astrophysics, 616(1).

Genet, R., Johnson, J., Buchheim, R., and Harshaw, R. (2015). Small Telescope Astronomical Research Handbook. Ed. Collins, D.

Harshaw, R. (2018). Gaia DR2 and the Washington Double Star Catalog: A Tale of Two Databases. Journal of Double Star Observations, 14(4):734740 .

Johnson, J., Genet, R., Armstrong, J., Boyce, G., Boyce, P., Brewer, M., Buchheim, R., Carro, J., Estrada, R., Estrada, C., et al. (2015). Astronomy Research Seminar. In Proceedings for the 34th Annual Conference of the Society for Astronomical Sciences, pages 139-148.

Korat, P., Beltzer-Sweeney, A., Vazquez, W., Correia, K., Calanog, J., Boyce, P., and Boyce, G. (2017). Astrometric Observations and Analysis of the Physical Binary Pair STF 296AB. JDSO, 13(4):529-533.

Mason, B., Wycoff, G., and Hartkopf, W. (2010). Washington Double Star Catalogue.

White, S., Gallegos, J., Boyce, G., Boyce, P., and Barnett, C. (2018). Near Infrared Robotic Observation of Double Star System WDS 13513-3928. Bulletin of the American Physical Society, 63. 